\section{In Pursuit of \\ Supersymmetry: Results from Lep 2}

\author{
Marc Besançon
}

Saclay

Our present understanding of the fundamental electroweak and strong interactions is formalized within the standard model (SM). Nevertheless the SM does not describe the gravitational interaction and suffers from several theoretical inconsistencies. Minimal supersymmetric extension of the SM (MSSM), predicting the existence of a spectrum of new particles, allows us to solve these problems. A report is given on the search for supersymmetric particles within the framework of the MSSM with conserved leptonic and baryonic numbers at Lep 2. No light supersymmetric particles have been found and constraints on the parameters of the MSSM are derived. The mass of the lightest supersymmetric particle is found to be greater than $22 \mathrm{GeV} / \mathrm{c}^{2}$.

In 1996 the large $e^{+} e$ collider Lep located at CERN in Geneva started its second phase, Lep 2, by running at centre of mass energies of $161 \mathrm{GeV}$ and $172 \mathrm{GeV}$. These

Fig $1 \mu, M_{2}$ excluded domain from neutralino and chargino searches, for $\tan \beta=2$. The particle masses, production cross-sections and decay branching ratios, are sensitive to these MSSM parameters, so the search is performed in all possible final states and the final result is best viewed in terms of these parameters. The grey regions are excluded at $95 \%$ confidence level

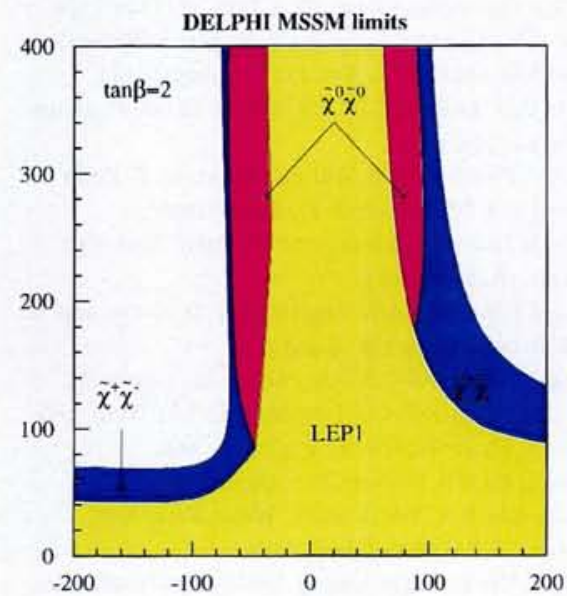

energy upgrades allowed us to continue the search for supersymmetry by exploring possible higher mass ranges for supersymmetric particles which have not been found in the previous phase of Lep (Lep 1), as well as in other experiments performed at Petra and SPS in the past or at the Tevatron, Pep and Hera (although two experiments, $\mathrm{H} 1$ and Zeus, earlier this year announced observation of events at very high Q2 which opens the way for speculations on their interpretations in terms of supersymmetry.)

In the framework of the standard model, the naturalness problem rises from the quadratic divergences in the Higgs boson mass correction. Introducing a new space-time symmetry, called supersymmetry, relating fermions and bosons, solves the naturalness problem and offers a framework for the description of the gravitational interaction. Inserting one supersymmetry operator in the SM leads to the extension of the particle spectrum. Each fundamental particle of the SM receives a so-called supersymmetric partner which differs in spin by $1 / 2$. For example, the electron with spin $1 / 2$ has a scalar ie spin o supersymmetric partner called the selectron. The spin 1 mediators of the electroweak forces (the gamma $\mathrm{W}$ and $\mathrm{Z}$ bosons) and strong forces (the gluons) have spin $1 / 2$ supersymmetric partners, the photino, wino, zino and gluinos - the suffix 'ino' is added to the name of the standard particle to derive its superpartner name. Furthermore, the simplest version of the supersymmetric extension of the SM requires the existence of two Higgs doublets, one coupling to the utype quark, the other to the d-type quark. These spin o Higgs fields have spin $1 / 2$ supersymmetric partners, the higgsinos.

Since no supersymmetric particles have been discovered so far, supersymmetry must be a broken symmetry.

Moreover, by solving the naturalness problem supersymmetric particles are expected to have masses of the order or less than $1 \mathrm{TeV}$. Several mechanisms have been proposed for supersymmetry breaking leading to different supersymmetric models. None of these models predicts the masses of the supersymmetric particles but allows us to express them in terms of restricted sets of parameters. One of the most popular and most developed model is the minimal supersymmetric extension of the standard model MSSM with its five generic parameters, among them $A, \mu, M_{2}, \tan \beta$, and $m_{\mathrm{o}}$ being, respectively, the Higgs to scalar tri-linear coupling, the Higgs mass parameter, the gauginos (photino, wino, zino and gluinos) mass parameter, the ratio of the Higgs vacuum expectation values and the mass parameter of the scalars. In this model, physical eigenstates, such as the four neutralinos $\left(\tilde{\chi}_{1,2,3,4,4}^{0}\right)$, are obtained from the mixing of photino, zino and neutral higgsinos or physical eigenstates such as the two charginos $\left(\tilde{\chi}_{1,2}^{ \pm}\right)$are obtained from the mixing of winos and charged higgsinos (table 1). Mixing the supersymmetric partners of the lefthanded and right-handed quarks allows us to build physical eigenstates for squarks. The two physical states of a squark ( $\tilde{q})$ may have different mass depending on the Yukawa coupling of the corresponding quark and on the MSSM parameters. For example, due to its heavy mass, the top quark $(t)$ Yukawa coupling is big and, in consequence, the two physical stops $\left(\tilde{t}_{1}, \tilde{t}_{2}\right)$, which are a mixing of the supersymmetric partners of the lefthanded top quark and the right-handed top quark, have very different mass, one being much heavier than the other, and

Fig 2 Excluded domains at the $95 \%$ confidence level in the plane of the right slepton (supersymmetric partner of right-handed lepton) mass (horizontal axis) and lightest neutralino mass (vertical axis). It is assumed that the slepton decays into a lepton and neutralino

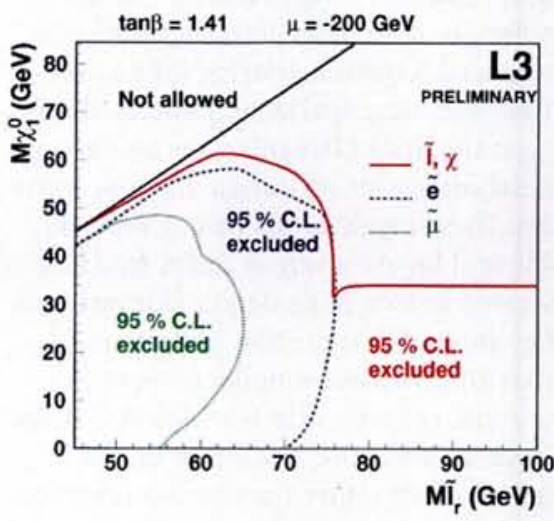


the lightest one being possibly lighter than the top quark. This phenomenon is particularly striking for the third family of quarks and holds also for the third family of charged leptons.

The conservation of the leptonic and baryonic numbers leads to a multiplicatively conserved quantum number, the so called $R$-parity $\left(R_{p}\right)$ which strongly determines the phenomenology for the MSSM since $R_{p}$ conservation tells us that supersymmetric particles are pair produced at colliders, that a supersymmetric particle decays always into another supersymmetric particle and a SM particle and finally that there exists a stable lightest supersymmetric particle (LSP). The first neutralino $\left(\tilde{\chi}_{1}^{\circ}\right)$ is at present the best candidate for being the LSP. The lack of knowledge concerning supersymmetry breaking forbids us to make any precise prediction on the masses of supersymmetric particles which, in consequence, have to be searched for at each energy upgrade of an accelerator, as is presently the case for Lep 2. A summary of the spectrum of the supersymmetric particles in the context of the MSSM is given in table 1. At Lep 2, the lightest $\tilde{\chi}_{1}^{ \pm}$, the two lightest $\tilde{\chi}_{1,2,3,4}^{0}$, ie $\tilde{\chi}_{1}^{\circ}$ and $\tilde{\chi}_{\tilde{\chi}_{2}}^{0}$ sleptons $(\tilde{l})$, and $\tilde{t}_{1}$ and sbottom $\left(\tilde{b}_{1}\right)$ have been

extensively searched for within the framework of the MSSM with conserved $R_{p}$ this is in the data of the four experiments Aleph, Delphi, L3 and Opal. In 1996, no events have been found in the Lep detectors which cannot get an interpretation in terms of SM processes seen as background processes. In the following some

Fig 3 Excluded neutralino mass $\left(M_{x}\right)$, domains at the $95 \%$ confidence level as a function of the $\tan \beta$ parameter. The lowest neutralino mass-limit of $22 \mathrm{GeV} / \mathrm{c}^{2}$ is considered an absolute lower bound of the lightest supersymmetric particle (LSP) mass regardless of other parameters

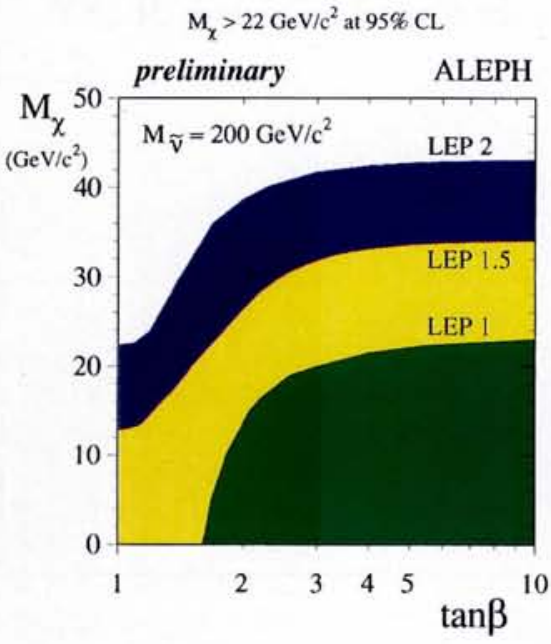

examples are given.

The lightest $\tilde{\chi}_{1}^{ \pm}$is searched for by assuming its decay into the LSP and a virtual $\mathrm{W}$ which subsequently decays into a pair of quarks or a lepton and neutrino. The LSP escapes the Lep detectors without interacting and is thus undetected. As far as energy measurements in the Lep detectors are concerned, and since they carry energy, outgoing and undetected LSP's will be a source of missing energy. Consequently, the signature of the pair production of the lightest $\tilde{\chi}_{1}^{ \pm}$can be either 4 jets or one lepton and 2 acoplanar jets or 2 acoplanar leptons with missing energy in each case - acoplanar jets are particles whose total momentum direction, projected onto the plane perpendicular to the beam axis, has an angle smaller than 180 degrees. The $\tilde{\chi}_{2}^{\circ}$ is assumed to decay into the LSP or $\tilde{\chi}^{\circ}{ }_{1}$ and a virtual $\mathrm{Z}$ which decays either into a pair of quarks or into a pair of leptons. The signature of the associated production of $\tilde{\chi}_{2}^{\circ}$ and $\tilde{\chi}_{1}^{\circ}$ consists then of 2 acoplanar leptons and missing energy or 2 acoplanar jets and missing energy. The $\tilde{\chi}_{1}^{ \pm}$mass is excluded up to the 1996 Lep 2 kinematical limit for high $m_{0}$, and combining with the $\tilde{\chi}_{1}^{\circ}$ search, exclusion regions of the MSSM $\left(M_{2}, \mu\right)$ parameters are obtained as can be seen from fig 1 from the Delphi experiment for $\tan \beta=2$.

The $\tilde{l}_{\mathrm{s}}$, like the selectron ( $\left.\tilde{e}\right)$, the smuon $(\tilde{\mu})$ and the stau $(\tilde{\tau})$ are assumed to decay into $e+\mathrm{LSP}, \mu+\mathrm{LSP}$ and $\tau+\mathrm{LSP}$ respectively, giving rise to the 2 acoplanar leptons $(e, \mu$ or $\tau)$ and missing energy signature for the pair production of $\tilde{e}, \tilde{\mu}$ or $\tilde{\tau}$. Regions of the $\tilde{l}$ mass and LSP mass plane are excluded as can be seen from fig 2 from the $\mathrm{L}_{3}$ experiment. Combining the negative results from the $\tilde{\chi}_{1,2}^{ \pm}, \tilde{\chi}_{1,2,3,4}^{0}$ and $\tilde{l}$ searches as well as constraints from the Higgs mass, the Aleph experiment is able to extract a lower limit on the mass of the $\operatorname{LSP}\left(\tilde{\chi}^{\circ}{ }_{1}\right)$ of $22 \mathrm{GeV} / \mathrm{c}^{2}$ as can been seen from fig 3 . If the $\tilde{\chi}^{ \pm}$, and the sneutrino $\tilde{v}$ are heavier than the $\tilde{\tau}_{1}$, this $\tilde{\tau}_{1}$ is assumed to decay mostly into charm and LSPs giving rise to the signature labelled as 2 acoplanar jets and missing energy. Regions of the $\tilde{\tau}_{1}$ mass and LSP mass plane are excluded as can been seen from fig 4 from the OPAL experiment, for various mixing angles.

In conclusion, in 1996 no light supersymmetric particles showed up in the data of the four experiments installed at the Lep collider, which was running at centre of mass energies of $161 \mathrm{GeV}$ and $172 \mathrm{GeV}$. This result allows us to put lower mass limits on the supersymmetric part1cles as well as to exclude domains in the parameter space of the MSSM with $R_{p}$ conserved.

During this summer and autumn, Lep has benefited from a further upgrade in energy. And, considering in addition to the MSSM other possibilities, such as the breaking of $R$-parity or models based on gauge mediated supersymmetry breaking, will allow for the non-trivial but fascinating pursuit of the search for supersymmetry which may be a fundamental facet of the unification of all interactions. The ultimate answer may come from the LHC which will hopefully explore supersymmetric-particle masses up to the $\mathrm{TeV}$ range. If supersymmetry is not found at these masses we should look at alternative solutions to solve the naturalness problem of the SM such as compositeness or technicolour. Nevertheless, supersymmetry would still remain the most promising way to include gravitational interaction even though it would only show up at energy scales like the Planck mass scale which are, unfortunately, out of the reach of present and planned accelerators.

\section{References}

For supersymmetry, see H. Nilles Phys.Rep $110 \mathrm{C}$ (1984) 1

Figure credits: Michael Schmitt (Aleph), Klas Hultqvist (Delphi), Andre Sopczak (L3), Shoji Asai and Sachio Komamiya (both OPAL).

Fig 4 Excluded domains at the $95 \%$ confidence level in the plane of the stop mass (horizontal axis) and the lightest neutralino, LSP, mass (vertical axis), for two stop mixing angles $(\theta=0.98$, for which the stop decouples from the $Z$ boson and so in consequence the stop pair production is minimum, and $\theta=0$, the pure left stop (supersymmetric partner of the left handed top quark) case for which the stop pair production is maximum. The region less than $45 \mathrm{GeV}$ on the horizontal axis has been excluded by Lep 1, and the shaded area is the excluded region of the D0 experiment of the Tevatron at Fermilab

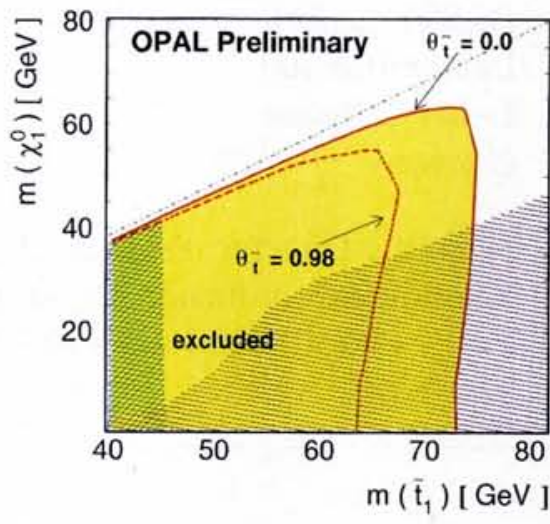

